\title{
Classification of Swarm Intelligence based Clustering Methods
}

\author{
Pooja Nagchoudhury \\ Department of Computer Science and Engineering \\ Rajasthan Technical University, \\ JIET, Jodhpur, Rajasthan, India
}

\author{
Kavita Choudhary \\ Department of Computer Science and Engineering \\ Rajasthan Technical University, \\ JIET, Jodhpur, Rajasthan, India
}

\begin{abstract}
The nodes in wireless sensor networks (WSN) need to be deployed optimally to cover whole geographic area and the communication link between them should be optimal. During deployment the proper strategy is required to optimally deploy the sensor nodes in the area. The sensor nodes can be compared with swarms which are homogenous agents. Swarm intelligence methods have been applied over the clustering of nodes in WSN and some of the approached have shown significant improvements in comparison with the non-swarm intelligence based algorithms. In this paper, we have tried to review latest techniques to create clusters using nature inspired methods. Proper classification with discussion of merits and demerits has been done. Scope of work in this area has been searched and inferred as conclusion. After the optimal deployment the next phase of needed is the energy efficient multi-hop routing among the nodes for communication of the packets to the base station. This multihop routing requires the optimal cluster head selection and maximum time of death of complete circuit achievable to get an optimally clustered network.
\end{abstract}

\section{Keywords}

Wireless sensor networks (WSN), Swarm Intelligence, Clustering, Bacterial Foraging Optimization (BFO) Algorithms, and Enhanced BFO.

\section{INTRODUCTION}

Wireless sensor network consist of several number of sensor nodes. Each sensor nodes are consisting of sensing unit, processing unit, energy unit and transmitting / receiving unit $[2,16,21]$. Components of wireless sensor networks are users, clusters, cluster head, base station and sensor nodes. All these components work together to perform the task. Rapid deployment, fault tolerance and self organize nature makes the WSN to use in many areas like in military surveillances, tracking application, climatic analysis, commercial applications etc [21]. While constructing WSN many issues must keep in consideration: reliability, flexibility, cost, easy of deployment etc $[4,18]$.

Data aggregation is important in WSN, for which the nodes in the field group together to form clusters. Clustering is required because of following reasons like Simplifies the task, reduce the communication overhead of the network, improves the life of network in WSN, avoid long distance data sensing and reduces collision in the network thus increases the throughput of the network [14, 24].

Bio-inspired methods: The biologically inspired model for WSN resolves some of the challenges of the WSN model. In biology the development of tissues give an inspiration for
WSN model, were the cells are first created in some area, then the cells are communicated by inter-cell signaling to form groups this process is known as lateral induction. Then among the groups lateral inhabitation takes place where some cell in the group perform task and rest of the cell in the group remain idle. This process is used in WSN model where the nodes in the network resemble the cells in the tissues $[12,14,16,19]$.

Routing of data from source to destination is important for fast and accurate delivery of data. Routing protocols of WSN are broadly divided in to two categories [26]:

i) On the bases of network structure

ii) On the bases of protocol operation

Single hop based routing approach is costly and consume more energy because the node far from the base station required more energy and time to send data to the base station. To overcome this problem of single hop routing approach the exchange of data between the nodes and the base station is carried out in multi hop manner. In which the node far from the base station instead of sending data directly to the base station send data to the neighborhood node and relay on node to send the data to the base station $[3,4,14,24]$.

\section{SWARM INTELLIGENCE}

A swarm is a group of number of simple and identical agents which cooperate with each other and environment to build up collective intelligent behavior to execute task required for their survival [27]. Swarm based algorithms recently became known as population based algorithms which are capable to provide solutions to several complex problems [5, 25, 27]. Swarm agents are interacting directly or indirectly. Swarm intelligent approach can applied in various problem domains which includes optimization problems, scheduling problems, data analysis and image processing, optimal routing etc [5]. Swarm intelligent models are particle swarm optimization, bacteria foraging etc [16]. Swarm intelligence has number of advantages, which are robustness, self-organization, flexibility and decentralization [27]. This makes swarm intelligence to use in distributed problem solving [25, 27].

\section{BACTERIA FORAGING ALGORITHM}

Bacterial foraging algorithms (BFA) copy the foraging nature of Escherichia coli bacteria (E.coli). E.coli bacterial finds the nutrient rich location in human intestine. The BFA models the behaviour of E.coli bacteria [2, 3, 16].

E.coli bacteria have two types of movements: swimming and tumbling. Swimming is the movement in which the bacteria moves in a straight line in a given direction, while in tumbling 
the bacteria randomly changes its direction of movement $[2$, $3,16]$.
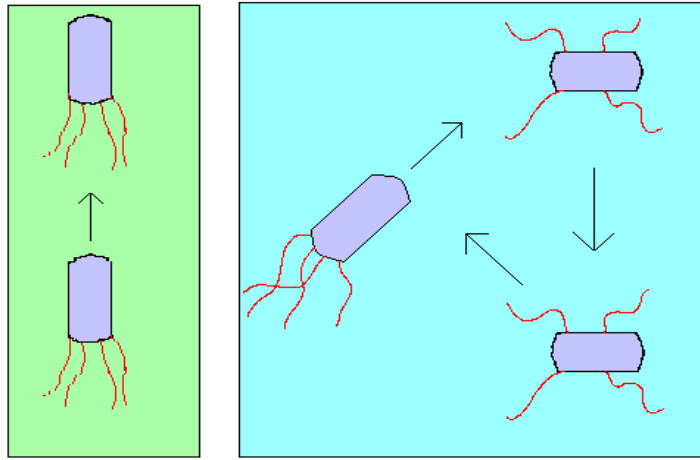

Fig 1. Swim and tumbling movement of bacteria

BFA have three steps:

$\begin{array}{ll}\text { a) } & \text { Elimination step } \\ \text { b) } & \text { Reproduction step. } \\ \text { c) } & \text { Chemotaxis step. }\end{array}$

The bacteria initially start with any random position. They evaluate the objective function to determine the movement in which direction. Then it moves in a particular direction again and evaluates the objective function. If it is better than the last one it continuously moves in the same direction until it gets the bad objective function. This whole step is chemotaxis step. After a specific number of chemotaxis steps the reproduction of the bacteria takes place in which the two bacteria combines to form a healthy bacteria and next is the elimination-dispersal step in which the bacteria splits into two bacteria and the non healthy bacteria is eliminated in this step. These steps are continuously repeated up to specific number of times $[2,34,16]$

\section{BASIC CLUSTERING}

The grouping of similar type of objects is called clustering. In WSN the nodes in the network are group together to form clusters. Each cluster in the network has a cluster head $[4,9$, $14,25]$. The cluster head of the cluster manage the members (nodes other than the cluster head) of the cluster. The nodes in the cluster transmits data to the cluster head, the cluster head performs various operations on the data received and sends the data to the other cluster head or the base station depends weather it is communicating directly with the base station or not. If it directly communicates with the base station it sends data directly to it otherwise, through multihop routing it sends the data to the base station $[4,24,21]$.

\section{CLUSTERING USING BFO}

The deployment of sensor node is done on the basis of clustering and BFA is used to find the accurate location of node [19]. The sensor node must cover the maximum points. The cluster is formed on the basis of centroid which is the mean of distance of all nodes which is lies in the range of the beacon node. The objective purpose is to decrease the distance between the beacon node and the other nodes. The information of each cluster is passing in the BFA to find the accurate location of sensor node within the cluster, so that the sensor node is placed in that position. Each node in the cluster run BFA to move towards the best position all nodes get collide at the best position satisfy the objective function to find the position, which is the position of beacon node(cluster head) of the cluster $[3,12,16]$.
How to localize a node using BFA:

a) Nodes are deployed in the sensor field. Some of them are beacon nodes and some are dumb nodes.

b) Localizable nodes are the nodes which are in the transmission range of the three or more non collinear nodes.

c) This node measures the distance between it and each of its neighboring nodes.

d) This localizable node runs the Bio-inspired algorithm separately or indecently to localize itself. They evaluate the objective function.

e) BFA search for the position of node that minimizes the objective function.

f) After all nodes determine their location, the difference is calculated between the actual node location and the location calculated by BFA which must be least.

g) The step is repeated until all the nodes find their position in the wireless sensor network $[16,19]$.

\section{CLASSIFICATION OF CLUSTERING BASED ON CH SELECTION METHOD}

On the basis of selection of cluster head the clustering algorithm is classified in various classes:

a) The use of identifier of nodes, where the smallest the identifier is elected as $\mathrm{CH}$. But this method have various disadvantages like selection is based on identifiers of nodes not on energy basis, requirement of unique identifier and assignment of identifier uniformly [24].

b) The use of degrees, where the $\mathrm{CH}$ is selected on the basis of number of neighbors. Higher the degree the probability to be the $\mathrm{CH}$ is more. It forms dense clusters, which creates non uniform size clusters, some $\mathrm{CH}$ has more overhead them others which causes high energy consumption [24].

c) The use of weight, the node with highest weight is selected as cluster head. The weight can be anything like the energy level, degree, and distance between neighbors or the combination of some parameters [24].

\section{ENHANCED BFO}

\subsection{Iterative Localization Algorithm:}

In iterative localization algorithm, the localization algorithm runs many time and in each new iteration all the settled nodes are now works as a beacon nodes and used to localize the unknown nodes [19]. Each nodes run its algorithm independently, the localization of node is not takes placed in centralized manner. Which increases the network traffic because all nodes sends their position to the centralized system and the power consumption of centralized system is more, which is now decreases if the localization algorithm runs in distributed manner [6, 19].

i. The nodes are randomly placed in the field; some know there position but rest of them identifies their position with the help of these nodes.

ii. When the unknown nodes falls in the communication range of three or more beacon nodes they get localized using the triangularization method.

iii. The nodes determine the distance between then and the neighboring beacon nodes.

iv. Now this unknown node runs the localization algorithm (BFA or PSO) independently.

v. The algorithm tries to find the best position of the nodes. 
vi. When all the nodes are localized the overall error is computed by determining the difference between the actual position of nodes and the position determine by the algorithm, which must be minimized.

vii. The above all steps are repeated until all the nodes are localized. In each iteration the settled node are now used as a beacon node to localize the unknown nodes $[6,18$, 19].

\subsection{Inter-Cell Communication Based:}

The author proposed a wireless sensor network model inspired by biology. The model works in three phases. The lateral induction phase, when the object appears near the node the nodes communicates to form compact cluster and select the nodes which have good observation quality and near to the node. Then the lateral inhabitation phase works in which the nodes were decided to be in the active or to be in inactive state depends on the energy level of the nodes.

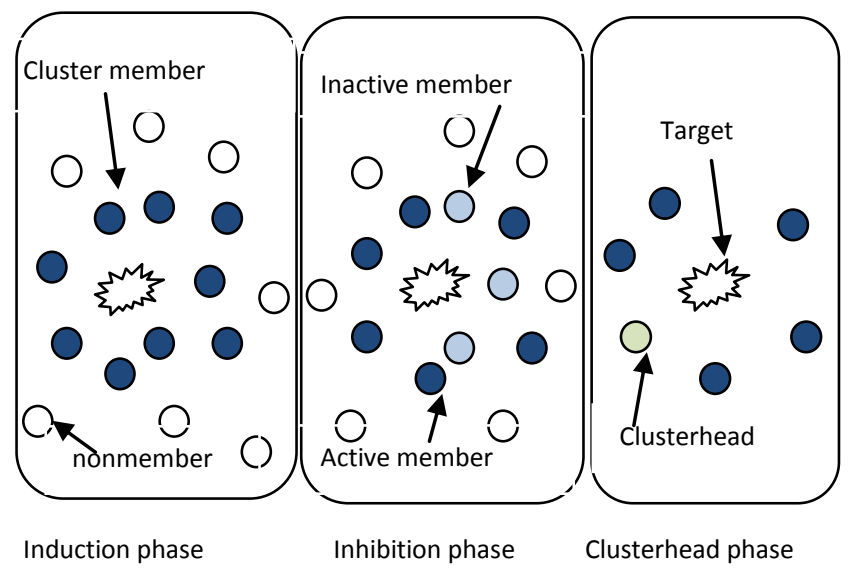

Fig 2. WSN model inspired by biology

Next in cluster head selection phase, the nodes in the cluster which are in the active state decide which node is going to be the head of the cluster. The cluster head have to perform many other tasks then the other nodes in the cluster. This requires more energy. The node with maximum energy level is selected as the head of the cluster; the nodes then construct the minimum spanning tree (MST) of the cluster to transmit the data from the active nodes to the cluster head. The cluster head then transmits the data to the base station [14].

\subsection{Basic Routing Algorithm Merging:}

Author proposed an algorithm which is the combination of BFA, LEACH and HEED. The three working phases of algorithm are:

i. The nodes in the cluster make use of BFA so that the distance between nodes in the cluster is maintained they are not too close and not too far. If they are close redundant data will received and if they are too far the consumption of energy increases.

ii. In the second phase, the cluster head is elected by using the LEACH and HEED protocol. By taken the probability of the node to be the cluster head and the energy of the node.

iii. In the third phase, the information is transmitted to the base station using the multiple-hop transmission. Were the information is sent to the neighboring Cluster head, then the neighboring cluster head decides the next receiver and finally to the base station. Instead of directly send it to the base station, which consumes more energy when it is far away from the base station [4].

Ad-hoc network is a network in which two or more mobile devices are interconnected to form a network. This is not based on the existing network requires minimum time to construct and with low cost [3]. The ad-hoc network is used in many areas of monitoring and tracking for which the devices must place in the proper position $[3,10]$. The BFA is used by the nodes to find the position in the network and form clusters for accurate working of the network $[3,16]$.

\subsection{Auxiliary Clusterhead (CH):}

A protocol of clustering which is advanced version of LEACH based on MIMO. It uses BFA for cluster formation and the enhancement is done by using the approach of multiple hop communication between the cluster head and base station and also considers the energy of the nodes to increase the life time of network. The algorithm selects the cluster head only when the node in the cluster dies because to minimize energy consumptions [9]. On the other hand in LEACH the selection of cluster head is takes place in every time which consumes more energy than the proposed algorithm [2]. The algorithm works on four phases [9]. Figure 3 show the model of auxiliary cluster head.

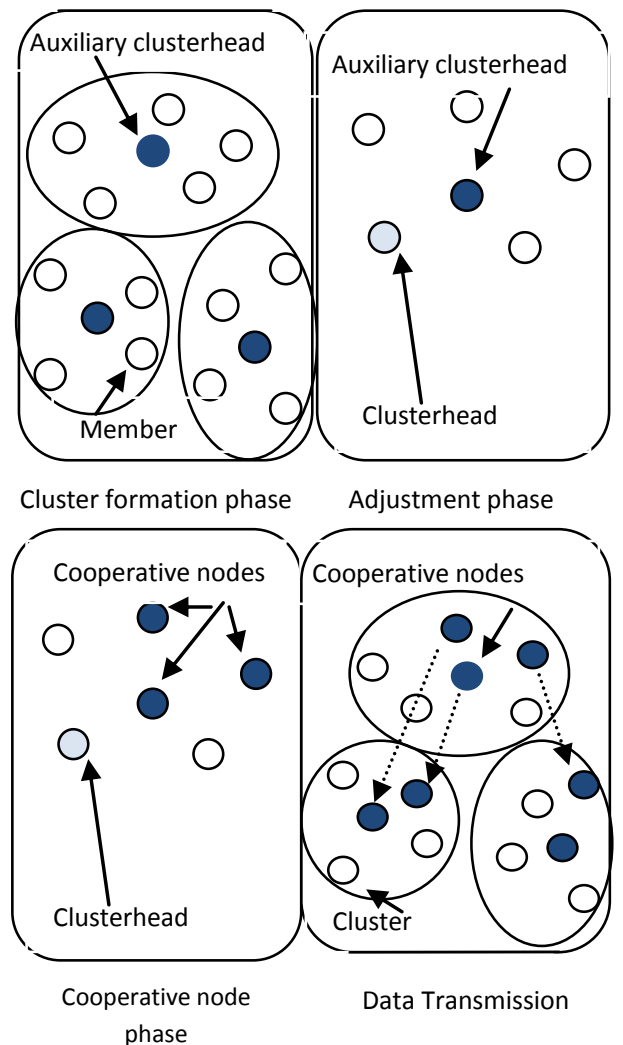

Fig 3. Auxiliary clusterhead model

i. Cluster formation phase, the base station selects some nodes as auxiliary cluster head ( $\mathrm{ACH})$ by considering the position of nodes. Then the base station divides the network in to the number of clusters as the number of $\mathrm{ACH}$. The base station selects the one ACH. The selected $\mathrm{ACH}$ selects the fixed number of nodes which is nearer to it as the member of cluster. The base station selects another ACH and the process continues till all the nodes 
are in cluster. If no nodes die the formation of cluster will stopped [3, 9].

ii. Cluster adjustment phase, the $\mathrm{ACH}$ select the actual cluster head by using the BFA and sends the information of it to all other cluster head and members of that cluster. The actual cluster head is selected on the basis of the node which has minimum displacement from its last position to final position [9].

iii. Selection of cooperative nodes, in this phase cooperative nodes are selected for MIMO communication, then cluster head sends the cooperative request to each cooperative nodes and then the receiving cooperative nodes sends the acknowledgement back to the cluster.

iv. Data transmission phase, the information are send using MIMO approach to the destination node [9].

\section{CLUSTERING BASED OTHER SWARM INTELLIGENCE METHODS}

\subsection{Genetic Algorithm:}

An algorithm which is an improvement of GA and based on filter replenishment strategy. Author describes how to get the region where the node must lie. The algorithm is suitable when there is less number of beacon nodes in the field and also in different size of wireless sensor network. It overcomes the problem of GA like global optimal solution and convergence. The algorithm works as [15]:

Step1: Find the region where the node must lie.

Step2: Generate randomly some nodes in that region.

Step3: Calculate the population divergence.

Step4: Apply filter and replenishment in the region to filter population.

Step5: Perform copy, crossover and mutation operation.

Step6: Perform the above steps until the number of iteration completed or the condition of convergence meet.

\subsection{Particle Swarm Optimization Algorithm (PSO):}

The PSO algorithm use in WSN to solve the various issues like for node deployment of stationary nodes, mobile nodes and base station, for localization of nodes, for energy efficient clustering and for data fusion [16]. PSO algorithm is modified according to solve the problem and each have its own optimization criterion [8]. PSO is a biological algorithm, while there are various traditional techniques are there to solve the problem but complexity of such techniques are increases exponentially as the size of problem is increased. But there is no such problem is there with biological algorithms. The traditional analytical techniques are slow or having less convergence for final result $[6,8,16]$. PSO algorithm is simple, has high convergence and finds accurate solution. The complexity of PSO is not increases as the size of the problem increases but it has some drawbacks like requirement of memory, requirement of distributed optimization frequently for data fusion $[5,6,8]$.The author extend an algorithm for node localization in field which is advance of PSO algorithm this improve version of PSO named HPSO (H-Best Particle Swarm Optimization) has high convergence them PSO. In HPSO the population of particles is divided in too small groups each group has local best position of particle among the entire particle in the group including the best position of the particle in all dimension and the overall best position of the particle as in PSO and compute the new position of the particle which is better from its previous $[6,16]$. The CLPSO (Comprehensive learning particle swarm optimization) determine velocity and position of particle in each dimension and generate indices of particles, which takes the value for each dimension from the pbest of particle. The indices take the value of $i$ with Pci known as learning probability. Each particle generates an arbitrary value. If this value is less than the Pci the dimension of particle learn from other pbest particle which is chosen randomly otherwise learn from its own pbest value. The PSO with respect to CLPSO has convergence but less accurate localization while the CLPSO have slow convergence but give more accurate localization result [5].

\section{DISTANCE ESTIMATION BASED}

The distance estimation algorithm is used to calculate the distance, the unknown distance of node with respect to some known distance and there received signal strength of these distance $[15,18]$.

Algorithm for three categories of sensor nodes localization:

\subsection{For Class-I}

Step 1: For each head node (beacon node) where $\mathrm{i}=1$ to $\mathrm{N}(\mathrm{N}$ is total number of head nodes)

Step 2: Calculate the distance between head node and there neighboring head node.

Step 3: For each head node select two nearest head nodes.

Step 4: Find out the node which lies in the range of three head nodes known as settled node.

Step 5: Then trilateration method is apply to determine the sensor nodes.

Step 6: Store the position.

Step 7: Repeat step 2 to 6 until all nodes gets identified.

\subsection{For Class-II}

Step 1: For each head node (beacon node) where $\mathrm{i}=1$ to $\mathrm{N}(\mathrm{N}$ is total number of head nodes)

Step 2: Calculate the distance between head node and there neighboring head node.

Step 3: For each head node select one nearest head node.

Step 4: Find out the node which lies in the range of two head nodes known as settled node.

Step 5: Then bounded method is apply to determine the sensor nodes.

Step 6: Store the position.

Step 7: Repeat step 2 to 6 until all nodes gets identified.

\subsection{For Class-III}

Step 1: For each head node (beacon node) where $\mathrm{i}=1$ to $\mathrm{N}(\mathrm{N}$ is total number of head nodes).

Step 2: Find out the node which lies in the range of only one head node known as settled node.

Step 3: Calculate the distance between the single head node and nodes nearer to it.

Step 4: Store the position.

Step 5: Repeat step 2 to 4 until all nodes gets identified

\section{GPS FREE}

Placing GPS system in each node to identify the position increases the cost of the WSN which is not possible. Some of the nodes in the WSN have GPS system these nodes are called head nodes, rest of the nodes were localized using these nodes. The head get information from the other sensor nodes, perform number of calculation for identify the position and sends the resulted information to the base station $[10,15,18]$. 
The vehicle mobile Ad hoc network is combining with WSN in various applications like finding the position of vehicle, speed of vehicle, sending alarm message of accident etc. Various algorithms are there which are based on GPS and non-GPS systems. Non-GPS system is preferable, which overcome the disadvantage of GPS systems and works well [18]. Some of them are Map Matching, which finds the position of vehicle and matches it with the stored map. Dead Reckoning, finds the position on the basis of time, speed, direction etc. The Cellular localization uses the cellular mobile infrastructure to find the position using triangulation method.

The new GPS-free algorithm is presented by author. In which author uses two types of nodes, one are stationary nodes which are known as RSS (Road side sensor) and other (sensor) are installed in the vehicles. The vehicle sends the request packet to the RSS to find the position and other vehicles. The RSS reply for the request packet to locate the vehicle. The vehicle gets the distance from three reply packets to locate accurately. Some of the node also retransmitted the request packet to get the data depends on the distance of the node to the base station. If all the RSS work properly the position of the vehicle is located accurately [10].

\section{MAJOR ADVANTAGES}

There are various analytical method are there for localization, node deployment, position of cluster head etc in WSN but the computational complexity of analytical methods are increases as the size of the problem increases. To overcome this biological algorithms are used in WSN $[12,14,16,19]$. There are various biological algorithms are there like PSO, BFA etc $[16,19]$. Among all these algorithms the BFA give the fast and accurate result for WSN [19]. Clustering is beneficial to transmit data to the base station because it decreases the network communication overhead, increases WSN life time, decreases collision in the network, increases throughput of the network etc [14].

\section{DISDVANTAGES}

The existing protocol for network control, clustering techniques and algorithm for scheduling of nodes in the WSN not work in energy efficient manner and have scalability problem $[2,9]$. In traditional clustering techniques the nodes in the cluster are selected in the basis of quality of observation by which the node located far way are also selected as the member of cluster. The nodes which are far way consuming more energy to transmit data to the cluster head which decreases the lifetime of the node in the cluster [14]. LEACH protocol while constructing the network not considers the position of the nodes and not handles the energy management of network $[2,4,9,13]$.

\section{COMPARATIVE STUDY}

BFA determines the coordinates of node accurately but slowly then PSO [16]. Genetic algorithm (GA) have slow convergence rate and suffer from localization error in some of the nodes in the network [15]. An algorithm which is variation of LEACH not work in large scale WSN but finds the cluster head only when the node in the cluster die rather than finding the cluster hand in each round as in LEACH $[2,9]$. A WSN model inspired by biology selects the nodes as a member of cluster if it's neighbor is selected in the cluster otherwise not, which lead to the problem that the node if have good observation quality not selected as the member of the cluster [14].

\section{CONCLUSION}

This paper summarizes the contributions of the last year papers in the field of nature inspired clustering. The classification of various algorithms has been done and they are algorithmically defined for the reference of the readers interested to work in this area to create brisk basics. The paper outlined the working and the pros \& cons of various approaches. It also concluded that not only the bio inspired clustering but also the multi-hop routing can be a decent source of optimal clustering of nodes in WSN. Among all Bacteria foraging technique is superior in terms of overall efficiency and effectively used for clustering methods in WSN.

\section{REFERENCES}

[1] S. Indu, M. Shubham, C. Santanu, and B. Asok, "BioInspired Distributed Sensing Using a Self-Organizing Sensor Network", Journal of Engineering, Vol. 2013, Article ID 959430, 16 pages, 2013.

[2] S. Sribala,'Energy Efficient Routing in Wireless Sensor Networks Using Modified Bacterial Foraging Algorithm", IJREAT International Journal of Research in engineering and Advanced Technology, Vol.1, Issue 1, March 2013, ISSN: 2320-8791

[3] Aruna, G. Vikas, "Soft Computing Implementation for Mobile Ad-hoc Network Optimization Using Bacteria Foraging Optimization Algorithm", International Journal of Computer Science and Communication Engineering, Vol.2, Issue 2, May 2013, ISSN 2319-7080.

[4] S. Anikit, T. Jawahar, "An Energy Efficient Network Life Time Enhancement Proposed Clustering Algorithm for Wireless Sensor Networks", International Journal of Enhanced Reacherch in Management and Computer Application, Vol. 2,Issue 7, July 2013,ISSN: 2319-7471, pp.1-4.

[5] M.V. Ramesh, P.L. Divya, P. Rekha, R.V. Kulkarni, "Performance Enhancement in Distributed Sensor Localization Using Swarm Intelligence", Advances in Mobile Network, Communication and its Applications (MNCAPPS), 2012 International Conference on, vol., no., 1-2 Aug. 2012, pp.103-106.

[6] K. Anil, K. Arun, S.S. Jasbir, S. Satvir, "Computational Intelligence based algorithm for node localization in Wireless Sensor Networks", 6th IEEE International Conference Intelligent Systems (IS), Sofia, 6-8 Sept. 2012, pp. $431-438$.

[7] P.T.V. Bhuvaneswari, S. Karthikeyan, B. Jeeva, M.A. Prasath, "An Efficient Mobility Based Localization in Underwater Sensor Networks", Computational Intelligence and Communication Networks (CICN), 2012 Fourth International Conference on, vol., no., 3-5 Nov. 2012, pp.90-94.

[8] V.K. Raghavendra, K.V. Ganesh, "Particle Swarm Optimization in Wireless-Sensor Networks: A Brief Survey", Systems, Man, and Cybernetics, Part C: Applications and Reviews, IEEE Transactions on, Vol.41, no.2, March 2011, pp.262-267.

[9] K.C. Navroop, "Energy Balancing Intelligent Clustering Protocol for Wireless Sensor Network", Journal of Global Research in Computer Science, Vol.2, no.7, July 2011, ISSN-2229-371X. 
[10] P.M. Jalil, M.G. Rama, B.G. Praveen, A. Ehsan, "Total GPS-free Localization Protocol for Vehicular Ad Hoc and Sensor Networks (VASNET)", Computational Intelligence, Modelling and Simulation (CIMSiM), 2011 Third International Conference on , vol., no., 20-22 Sept. 2011, pp.388-393.

[11] W. Chunjuan, Y. Junjie, G. Yanjie, Z. Zhimei, "Clusterbased routing protocols in wireless sensor networks: A survey", Computer Science and Network Technology (ICCSNT), 2011 International Conference on, vol.3, no., 24-26 Dec. 2011, pp.1659-1663.

[12] S.G. Gurjot, S. Kanwaljit, S.D Balwinder, "Sensor node deployment using Bacterial Foraging Optimization", Recent Trends in Information Systems (ReTIS), 2011 International Conference on, vol., no., 2123 Dec. 2011, pp.73-76.

[13] L. Qiao, C. Lingguo, Z. Baihai, F. Zhun, "A low energy intelligent clustering protocol for wireless sensor networks", Industrial Technology (ICIT), 2010 IEEE International Conference on, vol., no., 14-17 March 2010, pp.1675-1682.

[14] C. Charalambos, C. Shuguang, "A biologically inspired networking model for wireless sensor networks", Network, IEEE, vol.24, no.3, May-June 2010, pp.6-13.

[15] Y. Gao, Y. Zhuang, T. Ni, K. Yin, T. Xue, "An improved genetic algorithm for wireless sensor networks localization", Bio-Inspired Computing: Theories and Applications (BIC-TA), 2010 IEEE Fifth International Conference on , vol., no., 23-26 Sept. 2010, pp.439-443.

[16] V.K. Raghavendra, K.V. Ganesh, "Bio-inspired Algorithms for Autonomous Deployment and Localization of Sensor Nodes", Systems, Man, and Cybernetics, Part C: Applications and Reviews, IEEE Transactions on, vol.40, no.6, Nov. 2010, pp.663-675.

[17] Y. Li-ying, Z. Jun-ying, W. Wen-jun, "Cluster Ensemble Based on Particle Swarm Optimization", Intelligent Systems, 2009. GCIS '09. WRI Global Congress on, vol.3, no., 19-21 May 2009, pp.519-523.

[18] Z.S. Saleh, E. Vinayak, C. Wei, M. Richard, "Localization strategies for large-scale airborne deployed wireless sensors", Computational intelligence in miulticriteria decision-making, 2009. mcdm '09. ieee symposium on, vol., no., March 30 2009-April 2 2009, pp.9-15.
[19] V.K. Raghavendra, K.V. Ganesh, X.C. Maggie, "Bioinspired node localization in wireless sensor networks", Systems, Man and Cybernetics, 2009. SMC 2009. IEEE International Conference on, vol., no., 11-14 Oct. 2009, pp.205-210.

[20] Z. Bojkovic and B. Bakmaz, "A survey on wireless sensor networks deployment", WSEAS Trans. Commun., vol. 7, no. 12, 2008, pp. 1172-1181.

[21] N. M. A. Latiff, C. C. Tsimenidis, and B. S. Sharif, "Performance comparison of optimization algorithms for clustering in wireless sensor networks", in Proc. IEEE Int. Conf. Mobile Ad Hoc Sens. Syst., Oct. 8-11, 2007, pp. 1-4.

[22] G. Mao, B. Fidan, and B. D. O. Anderson, "Wireless sensor network localization techniques", Comput. Netw., vol. 51, no. 10, 2007, pp. 2529-2553.

[23] N. M. A. Latiff, C. C. Tsimenidis, and B. S. Sharif, "Energy-aware clustering for wireless sensor networks using particle swarm optimization", in Proc. 18th IEEE Int. Symp. Pers., Indoor Mobile Radio Commun., 2007, pp. 1-5.

[24] O. Younis, M. Krunz; S. Ramasubramanian, "Node clustering in wireless sensor networks: recent developments and deployment challenges", Network, IEEE, vol.20, no.3, May-June 2006, pp.20-25.

[25] C. Zhuo, M. Qing-Chun, "An incremental clustering algorithm based on swarm intelligence theory", Machine Learning and Cybernetics, 2004. Proceedings of 2004 International Conference on, Vol.3, no., 26-29 Aug. 2004, pp.1768-1772.

[26] J.N. Al-Karaki, A.E. Kamal, "Routing techniques in wireless sensor networks: a survey", Wireless Communications, IEEE, Vol.11, no.6, Dec. 2004, pp.628

[27] B. Wu, Z. Shi, "A clustering algorithm based on swarm intelligence", Info-tech and Info-net, 2001. Proceedings. ICII 2001 - Beijing. 2001 International Conferences on , vol.3, no., vol.3, 2001, pp.58-66.

[28] S. Kazem, M. Daniel, Z. Taieb, "Wireless Sensor Networks Technology, Protocols and Applications", A John Wiley and Sons, INC., Publication, 2007, ISBN 978-0-471-74300- 